\title{
Prevalencia de síntomas depresivos e invarianza factorial de la Escala de Depresión del Centro de Estudios Epidemiológicos (CES-D) en población indígena mexicana
}

\author{
Karen Lizbeth Franco-Díaz', Julián Alfredo Fernández-Niño2,3, Claudia Iveth Astudillo-García ${ }^{4}$ \\ 1 Escuela de Salud Pública de México, Cuernavaca, México \\ 2 Departamento de Salud Pública, Escuela de Medicina, Facultad de Salud, Universidad Industrial de Santander, \\ Bucaramanga, Colombia \\ 3 Departamento de Salud Pública, Universidad del Norte, Barranquilla, Colombia \\ 4 Servicios de Atención Psiquiátrica, Secretaría de Salud, Distrito Federal, México
}

Introducción. La versión breve de la Escala de Depresión del Centro de Estudios Epidemiológicos (CESD) es un recurso factible para la tamización de los síntomas de depresión en la población general, pero no se ha reportado la prevalencia en la población indígena, ni su invarianza factorial en Latinoamérica. Objetivo. Describir la prevalencia de los síntomas de depresión y la invarianza factorial de la versión breve de la escala CES-D en población indígena mexicana.

Materiales y métodos. Se hizo un estudio transversal en una muestra representativa de 37.165 adultos mexicanos de 20 a 59 años de edad. La identidad indígena se determinó mediante el propio reporte de la persona como hablante de una lengua indígena. Se conformaron ocho grupos de análisis según el sexo, el alfabetismo y el ser indígena. Se describió la prevalencia de los síntomas depresivos en cada grupo, así como la invarianza factorial de la configuración de los perfiles mediante un análisis factorial exploratorio. Las matrices de varianza y covarianza se compararon entre pares de perfiles usando el test modificado de Mantel.

Resultados. La prevalencia de síntomas depresivos en mujeres indígenas que sabían leer fue de $16,8 \%\left(\mathrm{IC}_{95 \%}: 13,4-20,3\right)$; en mujeres indígenas que no sabían leer, de $21,3 \%\left(\mathrm{IC}_{95 \%}: 15,5-27,1\right)$; en hombres indígenas que sabían leer de $8,5 \%\left(\mathrm{IC}_{95 \%}: 6,0-11,1\right)$, y en hombres indígenas que no sabían leer de $10,4 \%\left(\mathrm{IC}_{95 \%}: 5,2-15,6\right)$. No se encontraron diferencias significativas en las cargas factoriales entre los perfiles.

Conclusión. Se reportó una menor prevalencia de síntomas depresivos en indígenas que en la población no indígena. La escala CES-D en su versión breve mostró invarianza factorial al emplearla en la población indígena.

Palabras clave: población indígena; depresión; salud mental; análisis factorial; cultura; análisis multivariable.

doi: https://doi.org/10.7705/biomedica.v38i0.3681

Prevalence of depressive symptoms and factorial invariance of the Center for Epidemiologic Studies (CES-D) Depression Scale in a group of Mexican indigenous population

Introduction: The short version of the Center for Epidemiologic Studies (CES-D) Depression Scale is a viable tool for screening depressive symptomatology in the general population, but its factorial invariance for indigenous populations in Latin America has not been reported.

Objective: To describe the differences in the prevalence of depressive symptomatology and the factorial invariance of the short version of the CES-D scale for the Mexican indigenous population.

Materials and methods: We conducted a cross-sectional study with a representative sample of 37,165 Mexican adults aged 20 to 59 years. Indigenous identity was determined by self-report of being a speaker of an indigenous language. Eight groups of analysis were created according to gender, literacy, and being indigenous. An exploratory factor analysis was used to describe the prevalence of depressive symptoms as measured by the short version of the CES-D, as well as the configuration factorial invariance of the profiles. The variance-covariance matrices of the pairs of profiles were compared using the modified Mantel test.

\section{Contribución de los autores:}

Karen Lizbeth Franco-Díaz y Julián Alfredo Fernández-Niño: concepción del proyecto de investigación, análisis de los datos, diseño y redacción de la primera versión del manuscrito

Claudia Iveth Astudillo-García: interpretación de los datos, revisión crítica del contenido intelectual del manuscrito, ajuste y redacción de todas las versiones revisadas del manuscrito

Todos los autores aportaron a la discusión de resultados y a la redacción de la versión final del manuscrito. 
Results: The prevalence of depressive symptoms was $16.8 \%(95 \% \mathrm{Cl}: 13.4-20.3)$ for indigenous women who could read, $21.3 \%(95 \% \mathrm{Cl}: 15.5 \% 27.1)$ for indigenous women who could not read, $8.5 \%$ (95\% Cl: 6.0-11.1) for indigenous men who could read, and 10.4\% (95\% Cl: $5.2-15.6)$ for indigenous men who could not read. No significant differences in the factorial loads of the profiles were found.

Conclusion: The prevalence of depressive symptoms in indigenous people is lower than in the nonindigenous population. The factorial invariance of the CES-D scale was high for the indigenous population.

Key words: Indigenous population; depression; mental health; factor analysis,statistical; culture; multivariate analysis.

doi: https://doi.org/10.7705/biomedica.v38i0.3681

En el 2010, los trastornos depresivos representaban el $3 \%$ (rango: 2,2-3,8) del total de años de vida ajustados por discapacidad (AVAD) a nivel global, según los informes de la carga global de la enfermedad (1); sin embargo, se ha reportado que la población indígena tiene un mayor riesgo de problemas de salud mental en comparación con la población no indígena (2).

En el mundo hay alrededor de 300 millones de personas que pertenecen a pueblos indígenas, es decir, el 4,5\% de la población mundial; los datos de los últimos censos disponibles en Latinoamérica muestran que, en el 2010, había alrededor de 42 millones de personas indígenas, lo que representa casi el $8 \%$ de la población total, y países como México, Guatemala, Perú y Bolivia registraban las poblaciones más grandes, con más de $80 \%$ del total de la región (34 millones) (3).

La importancia de la atención en salud de los grupos vulnerables se reconoció mundialmente en el Foro Permanente para las Culturas Indígenas, en el cual se convocó a los gobiernos a mejorar los indicadores de los grupos indígenas, así como la recolección de datos que describieran su realidad según la etnia, el idioma, el sexo, la cultura o la adscripción de las propias personas a un grupo indígena determinado (4).

En este marco, la recopilación de datos fiables de salud mental mediante instrumentos de tamización adecuados, contribuye a enfrentar el problema de exclusión de los pueblos indígenas de los grandes temas de salud mental (5), ya que pueden presentarse síntomas depresivos específicos en esos grupos poblacionales, especialmente los relacionados con diferencias de orden sociocultural,

\footnotetext{
Correspondencia:

Julián Alfredo Fernández-Niño, Escuela de Medicina, Facultad de Salud, Universidad Industrial de Santander, Carrera $32 \mathrm{~N}^{\circ}$ 29-31, oficina 301, Bucaramanga, Colombia Teléfono: (577) 634 4000, extensión 3195 jafernandeznino@gmail.com

Recibido: 18/11/16; aceptado: 06/10/17
}

que podrían condicionar el diagnóstico (6), y una adecuada tamización podría constituir un paso inicial relevante en su contacto con los servicios de salud, por lo que la evaluación de los instrumentos estandarizados disponibles es indispensable.

México es un país multicultural, con más de 60 grupos indígenas y 69 lenguas y sus variantes (7), pero la información sobre la salud mental de dichos grupos en este país y en el continente es escasa (8). En este sentido, se ha reportado que la carga de la enfermedad en México presentaría diferencias en el grado de vulnerabilidad si el análisis se hiciera atendiendo la inclusión de los grupos indígenas (9). Por ello, y dada las altas prevalencias y la carga económica que representan, es necesario disponer de instrumentos adecuados para la medición de la depresión en estas comunidades $(10,11)$.

El instrumento de detección de sintomatología depresiva más utilizado en población general y en la investigación es la Escala de Depresión del Centro de Estudios Epidemiológicos (CES-D) (12), cuya aplicación masiva es viable, incluso en su versión breve $(13,14)$. Sin embargo, es necesario estudiar su óptima funcionalidad en diversos contextos, pues algunos estudios sugieren que su capacidad de detección podría variar dependiendo de la cultura (15-18) o del nivel de escolaridad (19), ya sea por una interpretación diferente de los ítems de la escala o por diferencias en la presentación clínica de la condición $(16,20)$.

Para comparar adecuadamente una condición entre grupos en contextos diferentes, se debe justificar su equivalencia formal y sustantiva, y esto está supeditado a la invarianza de medida de las puntuaciones (21), lo cual implica la revisión cualitativa del significado de los ítems, así como la evaluación cuantitativa de los diferentes niveles de la invarianza factorial y el funcionamiento diferencial de los ítems $(21,22)$.

La varianza factorial se puede definir como la consistencia de la estructura factorial de los ítems entre distintas subpoblaciones (22). Para 
establecer la invarianza factorial, se requieren pruebas de invarianza débil o de configuración, y de invarianza fuerte y estricta. La invarianza de configuración solo requiere de cargas factoriales invariantes; la invarianza factorial fuerte requiere, además, que las medias de los factores específicos (representados como 'interceptos' invariantes) también sean idénticos entre los grupos, en tanto que la invarianza factorial estricta exige que las varianzas residuales (las del factor específico más los errores de la variable) sean equivalentes entre los grupos (22).

Si bien la invarianza factorial de la escala CES-D se ha estudiado en diversas investigaciones cuyos objetivos incluían la exploración de la invarianza entre sexos (23), entre poblaciones social y culturalmente diferentes (18), y entre grupos que habían adquirido total o parcialmente una cultura nueva (24), entre otros, en el caso de la población indígena de México solo se ha explorado la versión de 20 ítems en el grupo ñahñú. Aunque en este grupo la escala mostró una buena consistencia interna (coeficiente alfa ordinal $=0,90$ ) y se obtuvieron tres factores que explicaban el 56,9 \% de la varianza, los autores concluyeron que la escala adaptada mostraba diferencias con la escala original debido a las diferencias culturales (25).

La utilización de los datos de la versión breve de la CES-D 7 empleada en la Encuesta Nacional de Salud y Nutrición 2012 en México (26), permitiría conocer la prevalencia de los síntomas depresivos en la población mexicana y analizar si presenta invarianza factorial de configuración en el contexto de los grupos indígenas, considerando variables como el sexo y el alfabetismo, las cuales pueden influir en el estado de salud mental de dicho grupos.

En este contexto, el objetivo del presente trabajo fue describir las diferencias en la prevalencia de los síntomas depresivos y la invarianza factorial de configuración de la versión breve de la escala CES-D en población indígena mexicana.

\section{Materiales y métodos}

\section{Diseño del estudio}

Se hizo un estudio de corte transversal a partir de los datos de la Encuesta Nacional de Salud y Nutrición (ENSANUT 2012), que se realiza en México cada seis años (27). Esta tiene un diseño muestral representativo a nivel nacional, estatal, y por estratos urbano y rural. Es una encuesta de múltiples fases, probabilística, diseñada para estimar la prevalencia de las condiciones de salud y de nutrición, y sus factores factores determinantes. La encuesta tiene un componente de salud y un componente de nutrición, y recopila datos de antropometría, seguridad alimentaria, tipo de sangre, nutrición y distribución de los alimentos. Es posible consultar información detallada sobre el diseño de la encuesta en los artículos publicados (26).

\section{Muestra de estudio}

La ENSANUT 2012 contó con una muestra de 50.528 viviendas, en cada una de las cuales se entrevistó, por lo menos, a un adulto de 20 años de edad o más. La muestra original incluyó a 46.277 adultos de 20 años o más, de los cuales 37.403 eran menores de 60 años. Se obtuvo información sobre las variables relevantes de 37.165 observaciones que constituyeron la muestra analítica del presente estudio; se excluyeron 218 por falta de información sobre síntomas depresivos y 20 que no tenían información sobre la característica de ser indígena.

Con la muestra analítica se definieron los siguientes ocho grupos de estudio, denominados como "perfiles", a partir de las variables que podrían introducir cambios en los resultados por estar relacionadas con las condiciones de vida de los participantes: sexo, alfabetismo y ser hablante de una lengua indígena:

1. Mujeres hablantes de lengua indígena que sabían leer

2. Mujeres no hablantes de lengua indígena que sabían leer

3. Mujeres hablantes de lengua indígena que no sabían leer

4. Mujeres no hablantes de lengua indígena que no sabían leer

5. Hombres hablantes de lengua indígena que sabían leer

6. Hombres no hablantes de lengua indígena que sabían leer

7. Hombres hablantes de lengua indígena que no sabían leer y

8. Hombres no hablantes de lengua indígena que no sabían leer

\section{Instrumentos de recolección de la información}

La ENSANUT 2012 contó con tres cuestionarios, a saber:

1) la encuesta individual con versiones para cada grupo de edad (menores de 0 a 9 años; adolescentes de 10 a 19 años; adultos de 20 años o más), en la cual se incluía el apartado de depresión correspondiente a la escala CES-D de siete ítems; 
2) la encuesta sociodemográfica del hogar (realizada al jefe de hogar) que recogía los datos de nivel socioeconómico $y$,

3) una encuesta de integrantes, en la cual se ampliaba la información sociodemográfica de los integrantes del hogar.

En este estudio, se utilizó la información de los adultos de 20 a 59 años de edad proveniente de estos tres cuestionarios. Los instrumentos pueden consultarse en línea (www.ensanut.org).

\section{Medición de la depresión}

Se utilizó la versión abreviada de 7 ítems de la escala en español del Centro de Estudios Epidemiológicos (CES-D), con un punto de corte óptimo estimado de 9 puntos en la población general, valores de sensibilidad de $90,2 \%$ y de especificidad de $86 \%$, un valor en la curva de la característica operativa del receptor (Receiver Operating Characteristic, ROC) de $88 \%$ utilizando el ICD-10, y de $80,4,89,6$ y $85 \%$, respectivamente, al utilizar el DSM-IV $(13,14)$. Por encima del punto de corte de 9 , se consideró que las personas tenían síntomas depresivos clínicamente significativos (13).

La escala fue utilizada por entrevistadores previamente capacitados por el área de Encuestas del Instituto Nacional de Salud Pública. Los entrevistadores debían leer la siguiente oración: "A continuación le mencionaré sentimientos o conductas que usted haya podido experimentar. Por favor, dígame con qué frecuencia se ha sentido así durante la última semana". Antes de iniciar la lectura de cada ítem debían decir "Durante la última semana...", y después, leer las opciones de respuesta y anotar solo una; la capacitación práctica se reforzó con 'sociodramas' y práctica de campo (27).

Cada ítem de la escala CES-D está construido con una escala de Likert que va de 0 a 3 puntos, donde 0 es la puntuación mínima que se puede obtener y 21 la máxima (14). El puntaje más alto de cada ítem indica mayor intensidad del síntoma depresivo, exceptuando al ítem número seis que se puntúa al contrario. La escala responde a las preguntas:

1) ¿Sentía como si no pudiera quitarse de encima la tristeza?

2) ¿Le costaba concentrarse en lo que estaba haciendo?

3) ¿Se sintió deprimido?

4) ¿Le parecía que todo lo que hacía era un esfuerzo?
5) ¿No durmió bien?

6) ¿Disfrutó de la vida?, y

7) ¿Se sintió triste?

La escala de Likert para cada respuesta tiene las opciones: "rara vez o nunca" (menos de un día), "pocas veces o alguna vez" (1 a 2 días), "un número de veces considerable" ( 3 a 4 días) y "todo el tiempo o la mayoría del tiempo" (5 a 7 días), y todas se refieren a los síntomas durante la última semana (13).

\section{Condición de pertenencia a un pueblo indígena}

Para establecer la identidad indígena, se creó la variable de ser hablante de una lengua indígena, definida como la respuesta afirmativa a la pregunta “¿Usted habla alguna lengua indígena?”; esto debido a las múltiples formas de identificación de las poblaciones indígenas, las cuales abarcan características de sexo, idioma, creencias y culturas ancestrales, así como la adscripción de las propias personas para diferenciarse de otras poblaciones indígenas $(28,29)$.

\section{Covariables}

Se consideraron en el análisis el sexo, el "estrato rural" definido para los participantes provenientes de localidades con menos de 2.500 habitantes, y el "estrato no rural" para quienes provenían de ciudades de 100.000 o más habitantes, o las zonas urbanas con localidades de 2.500 a 99.999 habitantes, según la definición de la ENSANUT 2102 (27), y, por último, el alfabetismo, el cual se reportó de manera dicotómica e incluyó a los participantes que sabían leer y escribir, definido con la pregunta “¿Usted sabe leer y escribir un recado?”.

\section{Análisis estadístico}

Se determinó la prevalencia de los síntomas de cada uno de los ocho perfiles previamente descritos, y se obtuvieron las frecuencias de respuestas positivas (positividad) de cada ítem de la escala CES-D, definida como la presencia de síntomas depresivos, por lo menos, durante un día en la semana anterior.

Con base en el puntaje de los síntomas depresivos, se estimó la prevalencia de aquellos clínicamente significativos usando el punto de corte de $\geq 9$ previamente validado para este grupo de edad (13). Para cada prevalencia se estimó el intervalo de confianza de $95 \%$, y las prevalencias entre grupos se compararon estadísticamente mediante pruebas de ji al cuadrado. Por último, se calculó el alfa ordinal de la escala a partir de 
la matriz policórica de los ítems. Esto último se hizo solamente para explorar preliminarmente su consistencia interna.

Invarianza factorial de configuración. Para la evaluación de la invarianza de configuración, se hizo un análisis factorial exploratorio de cada perfil definido a partir de la matriz policórica, para lo cual se empleó la escala de Likert. Para este análisis se hizo una extracción estándar utilizando un método de estimación de máxima verosimilitud y sin restricción preliminar de los factores esperados. En todos los subgrupos analizados (perfiles) se identificó únicamente un factor, pues los valores propios estimados a partir del segundo factor fueron muy pequeños (menores de 1). Dado que al identificarse una estructura unifactorial la solución es única, no se rotó ninguna de las matrices.

Como se explicó previamente, las cargas factoriales de la escala se estimaron para cada uno de los perfiles definidos, primero en toda la muestra y, posteriormente, por separado para los diferentes grupos (perfiles) de hablantes y no hablantes de una lengua indígena, aunque específicamente restringido a las áreas rurales. Se procedió así ya que se consideró que, en el análisis preliminar (de toda la muestra), la variable "ser hablante de una lengua indígena" podría estar ocultando un efecto diferencial entre áreas rurales y urbanas en la estructura factorial, al ser los indígenas una población culturalmente heterogénea. Lo que se quería explorar específicamente con este análisis era si el "efecto" de "ser hablante de una lengua indígena" sobre las cargas factoriales podría verse modificado por el estrato de urbanidad, dado que es un hecho bien reconocido que hay diferencias culturales y brechas socioeconómicas entre los indígenas y los no indígenas en México, que podrían determinar un comportamiento distinto frente a la escala y variarían entre el área rural y la urbana (5), pues, especialmente en las grandes ciudades, el proceso de incorporación y asimilación de los indígenas al mundo industrializado y a la cultura occidental puede ser más acentuado y, por ende, las diferencias entre indígenas y no indígenas no serían tan grandes en ellas como en el mundo rural (5).

Se compararon las matrices por perfil desde una perspectiva descriptiva y mediante la prueba estadística modificada de Mantel para comparación de matrices del mismo rango para cada par posible de matrices (30).

Debe aclararse que el test de Mantel compara las matrices de varianza y covarianza generadas por los valores propios, no por los valores propios per se. Sin embargo, si las matrices de varianza y covarianza son diferentes, lo serán también sus respectivos valores propios (obtenidos a partir de cada matriz mediante la misma técnica de extracción). En este caso, las matrices se generaron a partir del mismo número de determinantes (los ítems), así que lo que se comparó estrictamente fue la forma, entendiendo por esta su estructura de correlación reflejada en la matriz de varianza y covarianza. Como ya se mencionó anteriormente, para ello se utilizó el test modificado de Mantel.

El test de Mantel estándar usa la correlación de cada pareja de elementos en ambas matrices y las compara entre sí. En este caso, se compara cada varianza y cada covarianza de una matriz con su equivalente en la otra matriz, usando como hipótesis nula las diferencias esperadas por azar si las matrices fueran idénticas.

En síntesis, a diferencia del test clásico, el test modificado tiene como hipótesis nula la homogeneidad (más que la independencia) de matrices, si utiliza la diagonal de la matriz, y si permite comparar varianzas y covarianzas más que correlaciones. El test se aplicó para comparar todos los pares de matrices de acuerdo con el perfil. Dado que se trataba de comparaciones múltiples, se usó la corrección de Bonferroni para penalizar los valores de $\mathrm{p}$ según el número de comparaciones.

Todos los análisis descriptivos consideraron el diseño muestral poliétapico y estratificado por área (urbana o rural) y por Estado, así como por conglomerados en los que la unidad primaria de muestreo fueron las viviendas. En el análisis se especificaban la unidad primaria de muestreo, el estrato y el factor de expansión para cada sujeto, los cuales ya se habían calculado previamente para todos los participantes $(26,27)$.

Todas las estimaciones se realizaron con el programa Stata $14^{\mathrm{TM}}$ (Stata Corp, College Station, TX, USA), exceptuando el test modificado de Mantel, el cual se hizo en el programa R (Project for Statistical Computing).

\section{Consideraciones éticas}

El presente estudio es un análisis secundario a partir de la ENSANUT 2012, cuya base de datos es pública y puede utilizarse con fines de investigación, previa solicitud. El estudio original contó con la aprobación del Comité de Ética del Instituto Nacional de Salud Pública de México. Todos los participantes dieron su consentimiento informado. 


\section{Resultados}

Del total de la muestra analítica (37.165 observaciones), 57,8 \% correspondía a mujeres con una mediana de edad de 37 años (rango intercuartílico, $\mathrm{Rl}=29-46)$; la mediana de edad para hombres fue de $38(\mathrm{RI}=29-47)$. El $93 \%$ de la población general sabía leer y, entre la población hablante de una lengua indígena, el $78 \%$. En el cuadro 1 se muestra la distribución de estas características para los ocho perfiles.

Los resultados de los cuadros se presentan de tal manera que su lectura permite una comparación entre observaciones de la positividad de los ítems de la escala CES-D en la población hablante de una lengua indígena y en la población no hablante del mismo sexo y estatus de alfabetismo.

En el cuadro 2 se evidencia que los cuatro perfiles con mayor prevalencia de síntomas depresivos clínicamente significativos, correspondieron a las mujeres $(p<0,001)$. La prevalencia en mujeres fue el doble que en hombres del mismo perfil (hablantes de una lengua indígena que sabían leer): en mujeres hablantes de lengua indígena que sabían leer fue de $16,8 \%\left(\mathrm{IC}_{95 \%}: 13,4-20,3\right)$; en mujeres no hablantes de una lengua indígena que sabían leer fue de $18,1 \%\left(\mathrm{IC}_{95 \%}: 17,1-19,2\right)$; en mujeres hablantes de una lengua indígena que no sabían leer fue de $21,3 \%\left(\mathrm{IC}_{95 \%}: 15,5-27,1\right)$, y en mujeres no hablantes de una lengua indígena que no sabían leer fue de $28,1 \%\left(\mathrm{IC}_{95 \%}: 24,1-32,1\right)$. En ellas se observaron diferencias estadísticamente significativas entre el porcentaje de positividad de los síntomas depresivos clínicamente significativos tanto si sabían leer como si no, o si eran hablantes de una lengua indígena, siendo el grupo de no hablantes de una lengua indígena que no sabían leer el de mayor porcentaje de positividad.

En los grupos de hombres, la prevalencia de síntomas depresivos clínicamente significativos fue la siguiente: en hablantes de una lengua indígena que sabían leer, de 8,5\% (IC ${ }_{95 \%}$ : 6,6-11,1); en no hablantes de una lengua indígena que sabían leer, de 7,3\% ( $\left.\mathrm{IC}_{95 \%}: 6,6-8,1\right)$; en hablantes de una lengua indígena que no sabían leer, de $10,45 \%\left(I_{95 \%}: 5,2-15,6\right)$, y en no hablantes de una lengua indígena que no sabían leer, de 15,2\% $\left(\mathrm{IC}_{95 \%}: 10,4-20,0\right)$. No se reportaron diferencias estadísticamente significativas en la comparación entre quienes sabían leer y eran hablantes de una lengua indígena, o no, salvo en algunos ítems, especialmente entre quienes sabían leer.

En la figura 1 se presentan los gráficos de sedimentación que especifican los valores propios para cada perfil. En ellos se observó invariablemente un solo factor o componente importante en todos los perfiles, así como valores propios similares en todos ellos. En el análisis factorial se observó que en el perfil de las mujeres hablantes de una lengua indígena que sabían leer, el $71 \%$ de la varianza se explicó por el factor común, mientras que en el perfil de las mujeres no hablantes de una lengua indígena que sabían leer fue de $71,5 \%$, en las mujeres hablantes de una lengua indígena que no sabían leer fue de $73,1 \%$, y en las mujeres no

Cuadro 1. Datos sociodemográficos básicos de los ocho perfiles según sexo, alfabetismo y la condición de hablante de una lengua indígena en adultos mexicanos (20 a 59 años), ENSANUT 2012

\begin{tabular}{|c|c|c|}
\hline & Hombres $(42,1 \%)$ & Mujeres $(57,8 \%)$ \\
\hline & $n=15.625$ & $n=21.540$ \\
\hline & Mediana de edad: 38 años & Mediana de edad: 37 años \\
\hline & $\begin{array}{l}\text { Rango intercuartílico: } \\
\text { (29 a 47) }\end{array}$ & $\begin{array}{l}\text { Rango intercuartílico: } \\
\qquad(29 \text { a } 46)\end{array}$ \\
\hline Perfiles & n (\%) & n (\%) \\
\hline 1. Hombres hablantes de una lengua indígena que sabían leer & $1.177(5,3)$ & NA \\
\hline 2. Hombres no hablantes de una lengua indígena que sabían leer & $13.649(90,51)$ & NA \\
\hline 3. Hombres hablantes de una lengua indígena que no sabían leer & $197(0,9)$ & NA \\
\hline 4. Hombres no hablantes de una lengua indígena que no sabían leer & $602(3,3)$ & NA \\
\hline 5. Mujeres hablantes de una lengua indígena que sabían leer & NA & $1.407(4,6)$ \\
\hline 6. Mujeres no hablantes de una lengua indígena que sabían leer & NA & $18.606(89,7)$ \\
\hline 7. Mujeres hablantes de una lengua indígena que no sabían leer & NA & $563(1,7)$ \\
\hline 8. No hablantes de una lengua indígena que no sabían leer & NA & $964(4,0)$ \\
\hline
\end{tabular}

NA: no aplica

Proporciones ajustadas por el diseño muestral y calculadas sobre el total de cada sexo 
Cuadro 2. Porcentaje de positividad* (intervalo de confianza del $95 \%$ ) de cada ítem de la CES-D en población general de adultos mexicanos (20 a 59 años), ENSANUT 2012, considerando el diseño muestral

\begin{tabular}{|c|c|c|c|c|c|c|}
\hline \multirow[t]{2}{*}{ Ítems de la escala CES-D } & \multicolumn{2}{|c|}{ Población de hombres que sabían leer } & \multicolumn{4}{|c|}{ Población de mujeres que sabían leer } \\
\hline & $\begin{array}{l}\text { Hablantes de una } \\
\text { lengua indígena } \\
n=1.177\end{array}$ & $\begin{array}{c}\text { No hablantes de una } \\
\text { lengua indígena } \\
n=13.649\end{array}$ & $\mathbf{p}$ & $\begin{array}{l}\text { Hablantes de una } \\
\text { lengua indígena } \\
n=1.407\end{array}$ & $\begin{array}{c}\text { No hablantes de una } \\
\text { lengua indígena } \\
n=18.606\end{array}$ & $\mathbf{p}$ \\
\hline $\begin{array}{l}\text { 1. ¿Sentía como si no pudiera } \\
\text { quitarse de encima la } \\
\text { tristeza? }\end{array}$ & $\begin{array}{c}22,11 \\
(18.61-25,61)\end{array}$ & $\begin{array}{c}18,32 \\
(17,20-19,44)\end{array}$ & 0,101 & $\begin{array}{c}35,12 \\
(31,25-39,00)\end{array}$ & $\begin{array}{c}33,90 \\
(32,71-35,10)\end{array}$ & 0,740 \\
\hline $\begin{array}{l}\text { 2. ¿Le costaba concentrarse en } \\
\text { lo que estaba haciendo? }\end{array}$ & $\begin{array}{c}30,14 \\
(26,18-34,11)\end{array}$ & $\begin{array}{c}24,06 \\
(22,82-25,30)\end{array}$ & $<0,001$ & $\begin{array}{c}37,56 \\
(33,00-42,11)\end{array}$ & $\begin{array}{c}36,30 \\
(35,05-37,55)\end{array}$ & 0,399 \\
\hline 3. ¿Se sintió deprimido? & $\begin{array}{c}25,06 \\
(21,49-28,63)\end{array}$ & $\begin{array}{c}21,13 \\
(20,00-22,26)\end{array}$ & 0,046 & $\begin{array}{c}36,80 \\
(32,74-40,86)\end{array}$ & $\begin{array}{c}36,85 \\
(35,64-38,06)\end{array}$ & 0,179 \\
\hline $\begin{array}{l}\text { 4. ¿Le parecía que todo lo que } \\
\text { hacía era un esfuerzo? }\end{array}$ & $\begin{array}{c}32,58 \\
(28,29-36,88)\end{array}$ & $\begin{array}{c}24,86 \\
(23,58-26,14)\end{array}$ & $<0,001$ & $\begin{array}{c}39,12 \\
(34,74-43,51)\end{array}$ & $\begin{array}{c}35,01 \\
(33,75-36,27)\end{array}$ & 0,008 \\
\hline 5. ¿No durmió bien? & $\begin{array}{c}28,79 \\
(24,94-32,64)\end{array}$ & $\begin{array}{c}32,86 \\
(31,32-34,07)\end{array}$ & 0,026 & $\begin{array}{c}31,88 \\
(27,67-36,09)\end{array}$ & $\begin{array}{c}42,24 \\
(40,08-43,66)\end{array}$ & $<0,001$ \\
\hline 6. ¿Disfrutó de la vida?** & $\begin{array}{c}25,73 \\
(21,09-30,36)\end{array}$ & $\begin{array}{c}18,72 \\
(17,55-19,90)\end{array}$ & $<0,001$ & $\begin{array}{c}24,99 \\
(21,60-28,37)\end{array}$ & $\begin{array}{c}27,47 \\
(26,21-28,72)\end{array}$ & 0,446 \\
\hline 7. ¿Se sintió triste? & $\begin{array}{c}25,12 \\
(21,33-28,91)\end{array}$ & $\begin{array}{c}20,88 \\
(19,77-21,99)\end{array}$ & 0,193 & $\begin{array}{c}38,29 \\
(33,76-42,81)\end{array}$ & $\begin{array}{c}37,35 \\
(36,10-38,60)\end{array}$ & 0,337 \\
\hline $\begin{array}{l}\text { Presencia de síntomas } \\
\text { depresivos clínicamente } \\
\text { significativos }\end{array}$ & $\begin{array}{c}8,59 \\
(6,07-11,11)\end{array}$ & $\begin{array}{c}7,39 \\
(6,65-8,13)\end{array}$ & 0,571 & $\begin{array}{c}16,88 \\
(13,45-20,31)\end{array}$ & $\begin{array}{c}18,19 \\
(17,18-19,21)\end{array}$ & 0,047 \\
\hline \multirow{2}{*}{$\begin{array}{l}\text { Reactivos de la } \\
\text { escala CES-D }\end{array}$} & \multicolumn{3}{|c|}{ Población de hombres que no sabían leer } & \multicolumn{3}{|c|}{ Población de mujeres que no sabían leer } \\
\hline & $\begin{array}{c}\text { Hablantes de una } \\
\text { lengua indígena } \\
n=197\end{array}$ & $\begin{array}{c}\text { No hablantes de una } \\
\text { lengua indígena } \\
n=602\end{array}$ & $\mathbf{p}$ & $\begin{array}{l}\text { Hablantes de una } \\
\text { lengua indígena } \\
n=563\end{array}$ & $\begin{array}{c}\text { No hablantes de una } \\
\text { lengua indígena } \\
n=964\end{array}$ & $\mathbf{p}$ \\
\hline $\begin{array}{l}\text { 1. ¿Sentía como si no pudiera } \\
\text { quitarse de encima la } \\
\text { tristeza? }\end{array}$ & $\begin{array}{c}25,35 \\
(16,93-33,76)\end{array}$ & $\begin{array}{c}33,73 \\
(27,04-40,41)\end{array}$ & 0,276 & $\begin{array}{c}38,28 \\
(31,95-44,60)\end{array}$ & $\begin{array}{c}45,14 \\
(40,36-49,92)\end{array}$ & $<, 001$ \\
\hline $\begin{array}{l}\text { 2. ¿Le costaba concentrarse en } \\
\text { lo que estaba haciendo? }\end{array}$ & $\begin{array}{c}30,42 \\
(21,11-39,73)\end{array}$ & $\begin{array}{c}35,16 \\
(28,74-41,59)\end{array}$ & 0,523 & $\begin{array}{c}37,75 \\
(31,15-44,35)\end{array}$ & $\begin{array}{c}42,22 \\
(37,48-46,97)\end{array}$ & $<, 001$ \\
\hline 3. ¿Se sintió deprimido? & $\begin{array}{c}24,89 \\
(17,01-32,78)\end{array}$ & $\begin{array}{c}34,20 \\
(27,43-40,97)\end{array}$ & 0,443 & $\begin{array}{c}38,12 \\
(31,57-44,67)\end{array}$ & $\begin{array}{c}44,45 \\
(39,67-49,22)\end{array}$ & $<, 001$ \\
\hline $\begin{array}{l}\text { 4. ¿Le parecía que todo lo que } \\
\text { hacía era un esfuerzo? }\end{array}$ & $\begin{array}{c}37,27 \\
(26,11-48,43)\end{array}$ & $\begin{array}{c}38,59 \\
(32,33-44,86)\end{array}$ & 0,861 & $\begin{array}{c}46,61 \\
(40,44-52,77)\end{array}$ & $\begin{array}{c}44,37 \\
(39,65-49,09)\end{array}$ & $<, 001$ \\
\hline 5. ¿No durmió bien? & $\begin{array}{c}24,91 \\
(16,06-33,77)\end{array}$ & $\begin{array}{c}32,79 \\
(25,66-39,09)\end{array}$ & 0,393 & $\begin{array}{c}32,96 \\
(27,23-38,68)\end{array}$ & $\begin{array}{c}42,85 \\
(38,29-47,41)\end{array}$ & $<, 001$ \\
\hline 6. ¿Disfrutó de la vida?** & $\begin{array}{c}16,76 \\
(10,74-22,78)\end{array}$ & $\begin{array}{c}26,09 \\
(20,47-31,71)\end{array}$ & 0,027 & $\begin{array}{c}30,65 \\
(24,31-36,99)\end{array}$ & $\begin{array}{c}37,30 \\
(32,75-41,85)\end{array}$ & $<, 001$ \\
\hline 7. ¿Se sintió triste? & $\begin{array}{c}32,89 \\
(22,14-43,65)\end{array}$ & $\begin{array}{c}31,47 \\
(25,20-37,73)\end{array}$ & 0,875 & $\begin{array}{c}41,78 \\
(35,95-47,62)\end{array}$ & $\begin{array}{c}46,27 \\
(41,56-50,97)\end{array}$ & $<, 001$ \\
\hline $\begin{array}{l}\text { Presencia de síntomas } \\
\text { depresivos clínicamente } \\
\text { significativos }\end{array}$ & $\begin{array}{c}10,45 \\
(5,23-15,67)\end{array}$ & $\begin{array}{c}15,25 \\
(10,43-20,07)\end{array}$ & 0,374 & $\begin{array}{c}21,36 \\
(15,55-27,16)\end{array}$ & $\begin{array}{c}28,17 \\
(24,19-32,14)\end{array}$ & $<, 001$ \\
\hline
\end{tabular}

* Definida como presente durante, por lo menos, un día en la semana anterior.

** Por la naturaleza de la pregunta, el ítem 6 (¿Disfrutó de la vida?) se invirtió para su análisis.

Los valores de p corresponden a la diferencia de prevalencias entre hablantes y no hablantes de una lengua indígena con su par equivalente del mismo sexo y estatus de alfabetismo (Sí/No); estas diferencias se evaluaron estadísticamente usando pruebas de ji al cuadrado.

hablantes de una lengua indígena que no sabían leer fue de $71,8 \%$. En el caso de los hombres el porcentaje de varianza explicado por el factor común fue de $65,2 \%$, para los hombres hablantes de una lengua indígena que sabían leer, para los no hablantes de una lengua indígena que sabían leer fue de 66,6\%; en los hablantes de una lengua indígena que no sabían leer fue de $68,7 \%$, y para los no hablantes de una lengua indígena que no sabían leer fue de 69,4\%.
En el cuadro 3 se presentan los valores propios de cada perfil; se observó que las cargas factoriales más bajas fueron las de los ítems 5 ("No durmió bien") y 6 ("Disfrutó de la vida").

En el análisis secundario se consideraron los perfiles exclusivos de la población de áreas rurales (cuadro 4). Se encontró que la mayor prevalencia de síntomas depresivos clínicamente significativos se presentó, de nuevo, en las mujeres $(p<0,001)$. 


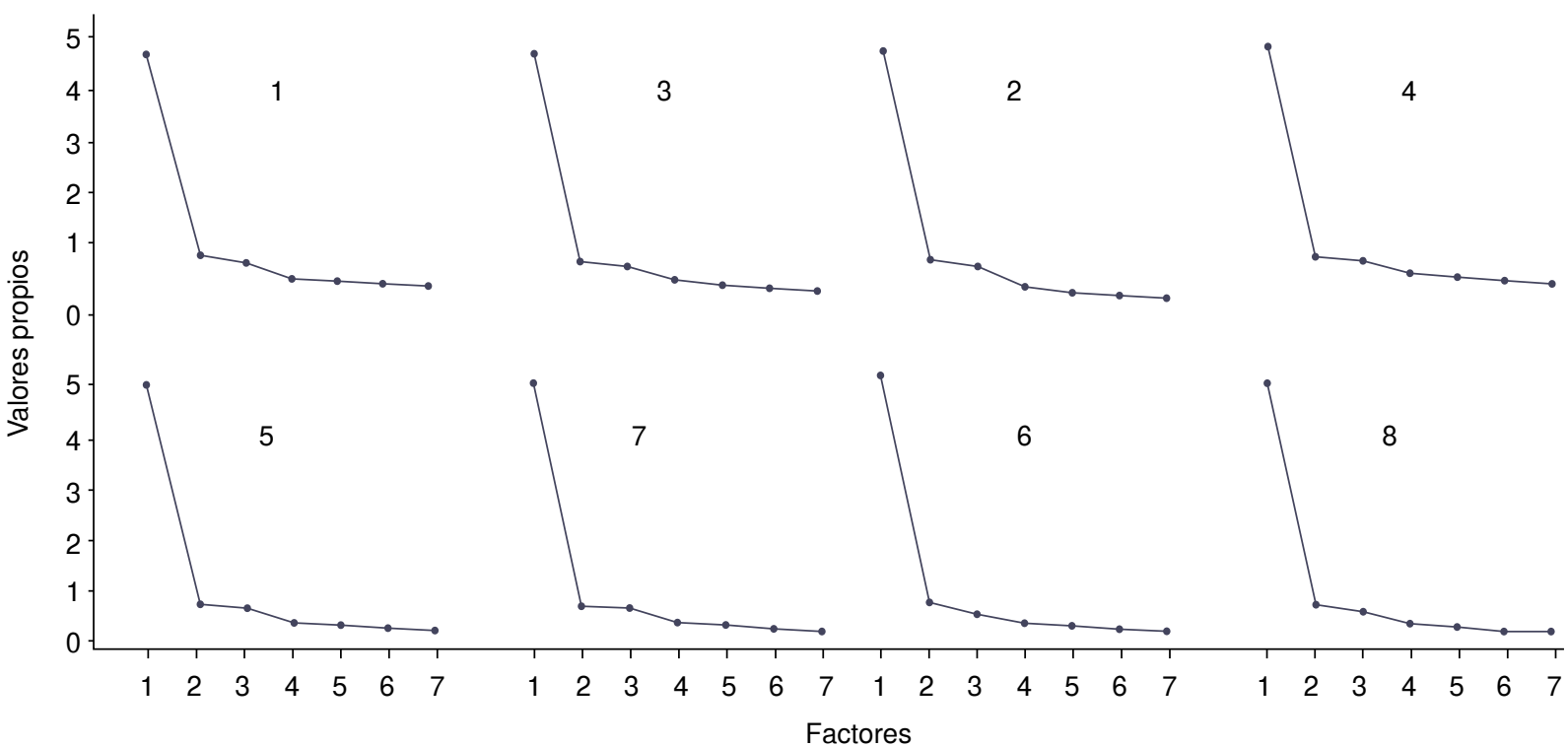

Figura 1. Gráficos de sedimentación de la escala CES-D según perfil de sexo, alfabetismo y condición de hablante de una lengua indígena en población general (20-59 años), ENSANUT, 2012

Perfiles de sexo, alfabetismo y condición de hablante de una lengua indígena en población general. 1: hombre hablante de una lengua indígena que sabía leer; 2 : hombre no hablante de una lengua indígena que sabía leer; 3 : hombre hablante de una lengua indígena que no sabía leer; 4: hombre no hablante de una lengua indígena que no sabía leer; 5: mujer hablante de una lengua indígena que sabía leer; 6 : mujer no hablante de una lengua indígena que sabía leer; 7: mujer hablante de una lengua indígena que no sabía leer; 8: mujer no hablante de una lengua indígena que no sabía leer. Cada perfil de hablante de una lengua indígena se presenta al lado de su par no hablante de una lengua indígena.

Cuadro 3. Cargas factoriales de los ítems de la escala CES-D según el perfil de sexo, estatus de alfabetismo y condición de hablantes de una lengua indígena en población general de adultos mexicanos (20 a 59 años), ENSANUT 2012*

\begin{tabular}{|c|c|c|c|c|}
\hline & $\begin{array}{l}\text { Población hablante de } \\
\text { una lengua indígena }\end{array}$ & $\begin{array}{l}\text { Población no hablante } \\
\text { de una lengua indígena }\end{array}$ & $\begin{array}{l}\text { Población hablante de } \\
\text { una lengua indígena }\end{array}$ & $\begin{array}{l}\text { Población no hablante } \\
\text { de una lengua indígena }\end{array}$ \\
\hline & \multicolumn{2}{|c|}{ Hombres que sabían leer } & \multicolumn{2}{|c|}{ Mujeres que sabían leer } \\
\hline Ítem & Factor 1 & Factor 1 & Factor 1 & Factor 1 \\
\hline 1 & 0,898 & 0,894 & 0,908 & 0,910 \\
\hline 2 & 0,872 & 0,882 & 0,895 & 0,890 \\
\hline 3 & 0,910 & 0,921 & 0,928 & 0,934 \\
\hline 4 & 0,852 & 0,833 & 0,887 & 0,875 \\
\hline 5 & 0,632 & 0,652 & 0,680 & 0,690 \\
\hline 6 & 0,520 & 0,584 & 0,667 & 0,681 \\
\hline 7 & 0,884 & 0,885 & 0,890 & 0,902 \\
\hline \multirow[t]{2}{*}{ Valor propio } & 4,57 & 4,67 & 4,97 & 5,01 \\
\hline & \multicolumn{2}{|c|}{ Hombres que no sabían leer } & \multicolumn{2}{|c|}{ Mujeres que no sabían leer } \\
\hline Ítem & Factor 1 & Factor 1 & Factor 1 & Factor 1 \\
\hline 1 & 0,912 & 0,901 & 0,916 & 0,911 \\
\hline 2 & 0,875 & 0,851 & 0,937 & 0,889 \\
\hline 3 & 0,956 & 0,929 & 0,929 & 0,934 \\
\hline 4 & 0,864 & 0,883 & 0,873 & 0,884 \\
\hline 5 & 0,637 & 0,716 & 0,777 & 0,744 \\
\hline 6 & 0,572 & 0,637 & 0,615 & 0,629 \\
\hline 7 & 0,909 & 0,873 & 0,896 & 0,897 \\
\hline Valor propio & 4,82 & 4,86 & 5,12 & 5,03 \\
\hline
\end{tabular}

* La comparación formal estadística se hizo con el test modificado de Mantel para cada par de matrices, sin encontrar ninguna diferencia estadísticamente significativa entre los pares de matrices comparados $(p>0,10)$. 
Cuadro 4. Porcentaje de positividad* (intervalo de confianza de $95 \%$ ) de cada ítem de la escala CES-D en adultos mexicanos (20 a 59 años) de áreas rurales, ENSANUT 2012, considerando el diseño muestral

\begin{tabular}{|c|c|c|c|c|c|c|}
\hline \multirow{2}{*}{$\begin{array}{l}\text { Ítems de la } \\
\text { escala CES-D }\end{array}$} & \multicolumn{2}{|c|}{ Población de hombres que sabían leer } & \multicolumn{4}{|c|}{ Población de mujeres que sabían leer } \\
\hline & $\begin{array}{c}\text { Hablante de una } \\
\text { lengua indígena } \\
\qquad n=720\end{array}$ & $\begin{array}{c}\text { No hablante de una } \\
\text { lengua indígena } \\
n=3,831\end{array}$ & $\mathbf{p}$ & $\begin{array}{c}\text { Hablante de una } \\
\text { lengua indígena } \\
\quad n=823\end{array}$ & $\begin{array}{c}\text { No hablante de una } \\
\text { lengua indígena } \\
n=5078\end{array}$ & p \\
\hline $\begin{array}{l}\text { 1. ¿Sentía como si no pudiera } \\
\text { quitarse de encima la tristeza? }\end{array}$ & $\begin{array}{c}19,94 \\
(18,70-22,60)\end{array}$ & $\begin{array}{c}26,17 \\
(23,99-28,35)\end{array}$ & 0,683 & $\begin{array}{c}30,93 \\
(26,56-35,29)\end{array}$ & $\begin{array}{c}36,38 \\
(33,98-38,78)\end{array}$ & 0,034 \\
\hline $\begin{array}{l}\text { 2. ¿Le costaba concentrarse en } \\
\text { lo que estaba haciendo? }\end{array}$ & $\begin{array}{c}31,19 \\
(26,36-36,02)\end{array}$ & $\begin{array}{c}26,17 \\
(23,99-28,35)\end{array}$ & 0,012 & $\begin{array}{c}37,56 \\
(33,00-42,11)\end{array}$ & $\begin{array}{c}39,88 \\
(37,23-42,53)\end{array}$ & 0,254 \\
\hline 3. ¿Se sintió deprimido? & $\begin{array}{c}23,59 \\
(19,48-27,70)\end{array}$ & $\begin{array}{c}24,77 \\
(22,60-26,94)\end{array}$ & 0,861 & $\begin{array}{c}33,51 \\
(28,21-38,82)\end{array}$ & $\begin{array}{c}40,36 \\
(38,09-42,62)\end{array}$ & 0,001 \\
\hline $\begin{array}{l}\text { 4. ¿Le parecía que todo lo que } \\
\text { hacía era un esfuerzo? }\end{array}$ & $\begin{array}{c}33,06 \\
(27,47-38,65)\end{array}$ & $\begin{array}{c}30,44 \\
(27,89-33,00)\end{array}$ & 0,327 & $\begin{array}{c}39,4 \\
(33,65-45,14)\end{array}$ & $\begin{array}{c}39,07 \\
(37,06-41,09)\end{array}$ & 0,975 \\
\hline 5. ¿No durmió bien? & $\begin{array}{c}23,33 \\
(18,64-28,02)\end{array}$ & $\begin{array}{c}29,36 \\
(27,13-31,60)\end{array}$ & 0,544 & $\begin{array}{c}29,77 \\
(24,44-35,09)\end{array}$ & $\begin{array}{c}39,35 \\
(37,22-41,49)\end{array}$ & $<0,001$ \\
\hline 6. ¿Disfrutó de la vida?** & $\begin{array}{c}23,84 \\
(19,24-28,44)\end{array}$ & $\begin{array}{c}20,62 \\
(18,44-22,81)\end{array}$ & 0,018 & $\begin{array}{c}27,29 \\
(22,64-31,94)\end{array}$ & $\begin{array}{c}31,18 \\
(28,71-33,66)\end{array}$ & 0,162 \\
\hline 7. ¿Se sintió triste? & $\begin{array}{c}24,42 \\
(19,55-29,30)\end{array}$ & $\begin{array}{c}23,27 \\
(21,35-25,19)\end{array}$ & 0,826 & $\begin{array}{c}36,59 \\
(31,66-41,51)\end{array}$ & $\begin{array}{c}40,79 \\
(38,36-43,25)\end{array}$ & 0,004 \\
\hline $\begin{array}{l}\text { Presencia de síntomas } \\
\text { depresivos clínicamente } \\
\text { significativos (SDCS) }\end{array}$ & $\begin{array}{c}8,3 \\
(4,87-11,73)\end{array}$ & $\begin{array}{c}8,84 \\
(7,51-10,18)\end{array}$ & 0,590 & $\begin{array}{c}14,42 \\
(10,57-18,28)\end{array}$ & $\begin{array}{c}19,71 \\
(17,73-21,69)\end{array}$ & 0,006 \\
\hline \multirow[t]{2}{*}{$\begin{array}{l}\text { Ítems de la } \\
\text { escala CES-D }\end{array}$} & \multicolumn{2}{|c|}{$\begin{array}{c}\text { Población de hombres que } \\
\text { no sabían leer }\end{array}$} & \multicolumn{4}{|c|}{$\begin{array}{l}\text { Población de mujeres que } \\
\text { no sabían leer }\end{array}$} \\
\hline & $\begin{array}{c}\text { Hablante de una } \\
\text { lengua indígena } \\
n=143\end{array}$ & $\begin{array}{l}\text { No hablante de una } \\
\text { lengua indígena } n=281\end{array}$ & p & $\begin{array}{c}\text { Hablante de una } \\
\text { lengua indígena } \\
n=402\end{array}$ & $\begin{array}{c}\text { No hablante de una } \\
\text { lengua indígena } \\
n=436\end{array}$ & $\mathbf{p}$ \\
\hline $\begin{array}{l}\text { 1. ¿Sentía como si no pudiera } \\
\text { quitarse de encima la tristeza? }\end{array}$ & $\begin{array}{c}35,64 \\
(25,30-45,97)\end{array}$ & $\begin{array}{c}34,75 \\
(26,39-43,12)\end{array}$ & 0,228 & $\begin{array}{c}33,06 \\
(26,41-39,71)\end{array}$ & $\begin{array}{c}53,18 \\
(46,55-59,81)\end{array}$ & $<, 001$ \\
\hline $\begin{array}{l}\text { 2. ¿Le costaba concentrarse en } \\
\text { lo que estaba haciendo? }\end{array}$ & $\begin{array}{c}30,42 \\
(21,11-39,73)\end{array}$ & $\begin{array}{c}36,23 \\
(28,74-43,64)\end{array}$ & 0,591 & $\begin{array}{c}34,47 \\
(27,21-41,73)\end{array}$ & $\begin{array}{c}50,03 \\
(43,30-56,76)\end{array}$ & $<, 001$ \\
\hline 3. ¿Se sintió deprimido? & $\begin{array}{c}33,75 \\
(23,81-43,70)\end{array}$ & $\begin{array}{c}34,51 \\
(26,99-42,02)\end{array}$ & 0,333 & $\begin{array}{c}31,19 \\
(24,60-37,77)\end{array}$ & $\begin{array}{c}55,66 \\
(48,55-62,77)\end{array}$ & $<, 001$ \\
\hline $\begin{array}{l}\text { 4. ¿Le parecía que todo lo que } \\
\text { hacía era un esfuerzo? }\end{array}$ & $\begin{array}{c}39,77 \\
(27,07-51,84)\end{array}$ & $\begin{array}{c}40,78 \\
(32,87-48,69)\end{array}$ & 0,812 & $\begin{array}{c}37,54 \\
(30,48-44,60)\end{array}$ & $\begin{array}{c}52,97 \\
(45,30-60,65)\end{array}$ & $<, 001$ \\
\hline 5. ¿No durmió bien? & $\begin{array}{c}28,18 \\
(18,43-37,94)\end{array}$ & $\begin{array}{c}29,64 \\
(22,33-36,95)\end{array}$ & 0,453 & $\begin{array}{c}30,87 \\
(23,85-37,97)\end{array}$ & $\begin{array}{c}45,32 \\
(39,88-50,76)\end{array}$ & $<, 001$ \\
\hline 6. ¿Disfrutó de la vida?** & $\begin{array}{c}21,71 \\
(13,75-29,67)\end{array}$ & $\begin{array}{c}32,08 \\
(25,03-39,13)\end{array}$ & 0,002 & $\begin{array}{c}29,96 \\
(22,69-37,26)\end{array}$ & $\begin{array}{c}44,09 \\
(38,36-49,81)\end{array}$ & $<, 001$ \\
\hline 7. ¿Se sintió triste? & $\begin{array}{c}30,79 \\
(21,20-40,37)\end{array}$ & $\begin{array}{c}34,25 \\
(26,73-41,78)\end{array}$ & 0,624 & $\begin{array}{c}34,47 \\
(28,59-40,35)\end{array}$ & $\begin{array}{c}51,25 \\
(44,54-57-96)\end{array}$ & $<, 001$ \\
\hline $\begin{array}{l}\text { Presencia de síntomas } \\
\text { depresivos clínicamente } \\
\text { significativos (SDCS) }\end{array}$ & $\begin{array}{c}14,63 \\
(7,24-22,02)\end{array}$ & $\begin{array}{c}16,87 \\
(9,62-24,11)\end{array}$ & 0,513 & $\begin{array}{c}16,42 \\
(10,77-22,07)\end{array}$ & $\begin{array}{c}32,72 \\
(25,74-39,69)\end{array}$ & $<, 001$ \\
\hline
\end{tabular}

* Definida como presente, por lo menos, durante un día en la semana anterior.

** Por la naturaleza de la pregunta, el ítem 6 (¿Disfrutó de la vida?) se invirtió para su análisis.

Los valores de $p$ corresponden a la diferencia de prevalencias entre hablantes y no hablantes de una lengua indígena con su par equivalente del mismo sexo y estatus de alfabetismo (Sí/No); estas diferencias se evaluaron estadísticamente usando pruebas de ji al cuadrado.

En cuanto a los hombres, el porcentaje de positividad de los ítems aumentó, especialmente en quienes no sabían leer. Al comparar los perfiles de hombres hablantes de una lengua indígena con los de los no hablantes, la positividad aumentó para los primeros, aunque dichas diferencias no fueron significativas. Se obtuvieron diferencias estadísticamente significativas en las mujeres al comparar su condición de hablantes de una lengua indígena con la de no serlo, siendo mayor la presencia de síntomas depresivos entre las no hablantes de una lengua indígena.

En el cuadro 5 se presenta la matriz de cargas factoriales por perfil en áreas rurales, en la cual se observa que no hubo diferencias por estrato. En las mujeres hablantes de una lengua indígena que sabían leer, 69,4\% de la varianza se explicó por el factor común: en no hablantes de una lengua 
Cuadro 5. Cargas factoriales de los ítems de la escala CES-D según el perfil de sexo, alfabetismo y condición de hablantes de una lengua indígena en adultos mexicanos (20 a 59 años) de estrato rural, ENSANUT 2012*

\begin{tabular}{|c|c|c|c|c|}
\hline & $\begin{array}{l}\text { Población hablante de una } \\
\text { lengua indígena }\end{array}$ & $\begin{array}{l}\text { Población no hablante de } \\
\text { una lengua indígena }\end{array}$ & $\begin{array}{l}\text { Población hablante de } \\
\text { una lengua indígena }\end{array}$ & $\begin{array}{l}\text { Población no hablante de } \\
\text { una lengua indígena }\end{array}$ \\
\hline & \multicolumn{2}{|c|}{ Hombres que sabían leer } & \multicolumn{2}{|c|}{ Mujeres que sabían leer } \\
\hline Ítem & Factor 1 & Factor 1 & Factor 1 & Factor 1 \\
\hline 1 & 0,876 & 0,887 & 0,904 & 0,904 \\
\hline 2 & 0,852 & 0,880 & 0,897 & 0,891 \\
\hline 3 & 0,908 & 0,917 & 0,919 & 0,933 \\
\hline 4 & 0,824 & 0,829 & 0,879 & 0,880 \\
\hline 5 & 0,694 & 0,656 & 0,660 & 0,700 \\
\hline 6 & 0,483 & 0,580 & 0,642 & 0,660 \\
\hline 7 & 0,867 & 0,880 & 0,880 & 0,896 \\
\hline \multirow[t]{2}{*}{ Valor propio } & 4,46 & 4,63 & 4,86 & 4,98 \\
\hline & \multicolumn{2}{|c|}{ Hombres que no sabían leer } & \multicolumn{2}{|c|}{ Mujeres que no sabían leer } \\
\hline Ítem & Factor 1 & Factor 1 & Factor 1 & Factor 1 \\
\hline 1 & 0,912 & 0,902 & 0,908 & 0,893 \\
\hline 2 & 0,875 & 0,818 & 0,945 & 0,882 \\
\hline 3 & 0,956 & 0,914 & 0,920 & 0,929 \\
\hline 4 & 0,864 & 0,864 & 0,869 & 0,865 \\
\hline 5 & 0,637 & 0,738 & 0,764 & 0,745 \\
\hline 6 & 0,572 & 0,598 & 0,632 & 0,605 \\
\hline 7 & 0,909 & 0,865 & 0,888 & 0,878 \\
\hline Valor propio & 4,84 & 4,72 & 5,09 & 4,88 \\
\hline
\end{tabular}

*La comparación formal estadística se hizo con el test modificado de Mantel para cada par de matrices, sin encontrar ninguna diferencia estadísticamente significativa entre los pares comparados $(p>0,10)$.

indígena que sabían leer, el 71,1\%; en hablantes de una lengua indígena que no sabían leer, el $72,7 \%$, y en no hablantes de una lengua indígena que no sabían leer, el 69,7\%; en tanto que para hombres hablantes de una lengua indígena que sabían leer la varianza explicó el 63,7\%; en los no hablantes de una lengua indígena que sabían leer, el 66,1\%; en los hablantes que no sabían leer, el $69,1 \%$, y en los no hablantes que no sabían leer, el 67,4\%. Asimismo, en los gráficos de sedimentación por perfil y áreas rurales (figura 2), se identificó solo un factor importante en todos los perfiles, y no se observaron diferencias en sus valores propios.

Por último, en el cuadro 6 se presenta la consistencia interna de la versión breve de la escala CES-D para cada perfil en la población general, la cual osciló entre 0,81 y 0,89, así como en áreas rurales, la cual osciló entre 0,798 y 0,887. La prueba estadística modificada de Mantel para la comparación de matrices en todos los pares de perfiles posibles, no arrojó diferencias en ninguno de ellos $(p>0,10)$.

\section{Discusión}

Hasta donde se sabe con base en lo publicado sobre tamización de problemas de salud mental en la población indígena de México, este es el primer análisis con datos representativos a nivel nacional.

El estudio permitió determinar la prevalencia de los síntomas de depresión, con énfasis en las potenciales diferencias en la población hablante de una lengua indígena. No se encontró una mayor prevalencia de síntomas en dicha población que en la población no hablante. Se exploraron, asimismo, las diferencias en la varianza factorial de la versión breve de la escala CES-D en la población hablante de una lengua indígena y en la no hablante considerando otras variables como el sexo, el estatus de alfabetismo y la pertenencia a áreas rurales, y se encontró que la escala se comportó de forma invariable en los diversos grupos de análisis.

En cuanto a la prevalencia de síntomas de depresión clínicamente significativos, la asociación más importante fue con el sexo, ya que estos se presentaron en los cuatro perfiles de mujeres, y fue más alta en mujeres no hablantes de una lengua indígena, datos que concuerdan con los estudios nacionales realizados en la población general $(13,31)$, en los cuales la presencia de estos síntomas ha sido significativamente mayor en las mujeres que no sabían leer. Este hallazgo coincide con los de otras investigaciones en las que el 


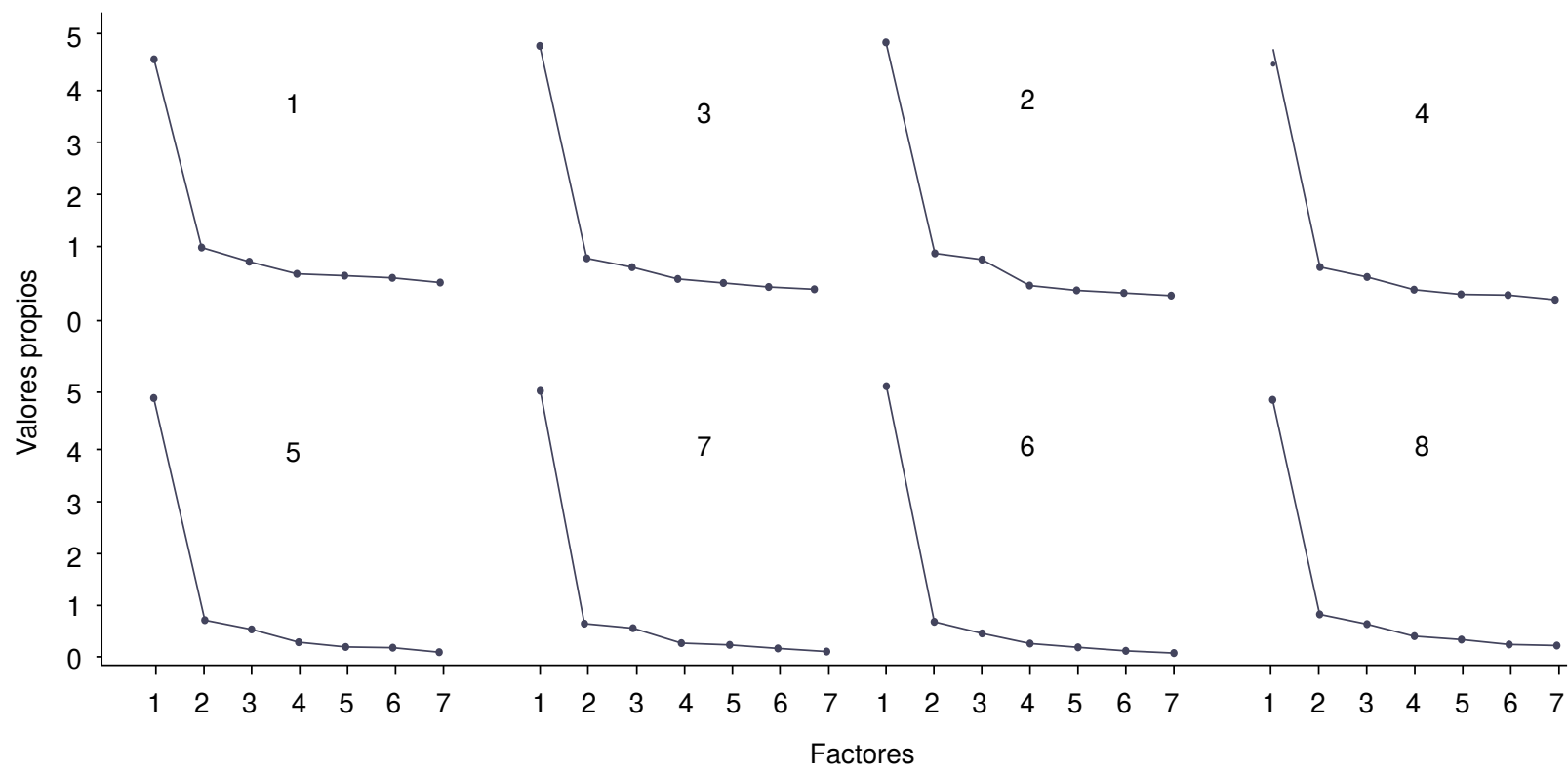

Figura 2. Gráficos de sedimentación de la escala CES-D según perfil de sexo, alfabetismo y condición de hablante de una lengua indígena en población de áreas rurales (20-59 años), ENSANUT, 2012

Perfiles de sexo, alfabetismo y condición de hablante de una lengua indígena en población general. 1: hombre hablante de una lengua indígena que sabía leer; 2 : hombre no hablante de una lengua indígena que sabía leer; 3 : hombre hablante de una lengua indígena que no sabía leer; 4: hombre no hablante de una lengua indígena que no sabía leer; 5 : mujer hablante de una lengua indígena que sabía leer; 6: mujer no hablante de una lengua indígena que sabía leer; 7: mujer hablante de una lengua indígena que no sabía leer; 8: mujer no hablante de una lengua indígena que no sabía leer. Cada perfil de hablante de una lengua indígena se presenta al lado de su par no hablante de una lengua indígena.

Cuadro 6. Alfa ordinal de la escala CES-D en población general y rural según perfil de sexo, alfabetismo y condición de hablantes de una lengua indígena en adultos mexicanos (20 a 59 años), ENSANUT 2012

\begin{tabular}{lcc}
\hline & $\begin{array}{c}\text { Población } \\
\text { hablante de } \\
\text { una lengua } \\
\text { indígena }\end{array}$ & $\begin{array}{c}\text { Población no } \\
\text { hablante } \\
\text { de una lengua } \\
\text { indígena }\end{array}$ \\
\hline $\begin{array}{l}\text { Población general } \\
\text { Hombres que sabían leer }\end{array}$ & 0,817 & 0,829 \\
Mujeres que sabían leer & 0,882 & 0,886 \\
Hombres que no sabían leer & 0,851 & 0,864 \\
$\quad$ Mujeres que no sabían leer & 0,892 & 0,893 \\
Población de área rural & & \\
Hombres que sabían leer & 0,799 & 0,831 \\
Mujeres que sabían leer & 0,870 & 0,887 \\
Hombres que no sabían leer & 0,853 & 0,845 \\
Mujeres que no sabían leer & 0,885 & 0,885 \\
\hline
\end{tabular}

tener bajo nivel educativo y ser mujer han sido factores psicosociales asociados con la presencia de depresión (32). También, se encontró que la presencia de los síntomas depresivos clínicamente significativos fue mayor en hombres hablantes de una lengua indígena que sabían leer que en los no hablantes que sabían leer; sin embargo, los datos fueron significativos únicamente para cinco de los siete ítems de la escala.
En otros estudios, los resultados han sido similares, por ejemplo, en un metaanálisis de Kisely, et al. (33), en el cual se comparó la prevalencia de los trastornos de depresión y la ansiedad en las poblaciones indígenas de las Américas con las de grupos no indígenas con características sociodemográficas similares, no hubo diferencias en las prevalencias en los 12 meses anteriores. En el caso de la prevalencia a lo largo de la vida, las tasas de ansiedad generalizada y de trastornos depresivos fueron significativamente inferiores en los participantes indígenas. Además, estos resultados fueron similares en el análisis de los grupos de Latinoamérica, Canadá y Estados Unidos, por lo que se concluyó que los factores de riesgo de las enfermedades psiquiátricas serían el resultado de una compleja interacción entre factores biológicos, educativos, económicos y socioculturales que puede variar según los trastornos.

Por otra parte, en el presente trabajo no se registró información significativa que indicara diferencias entre la población de las áreas rurales y la población general. En algunas investigaciones se ha encontrado que en la población indígena rural se presentan menos síntomas de tipo afectivo, especialmente en las mujeres, y es más común 
que reporten síntomas somáticos y de disforia (3437). En una investigación realizada en la población indígena del altiplano andino de Ecuador (38), también se observó una mayor prevalencia de manifestaciones somáticas de depresión que cognitivas. Sin embargo, en el presente estudio no se observó un patrón claro que diferenciara los síntomas de depresión entre los grupos.

En cuanto a la invarianza factorial de configuración, la descripción estratificada por perfil evidenció que la escala no variaba entre hablantes y no hablantes de una lengua indígena, independientemente del sexo y el alfabetismo. Asimismo, la invarianza se mantuvo en el análisis de los perfiles en áreas rurales, de manera que los resultados fueron consistentes, incluso en diferentes estratos de la población.

En otras investigaciones que han explorado la invarianza factorial mediante el análisis factorial confirmatorio, se han reportado diferencias entre grupos en la CES-D de 20 ítems. Posner, et al. (24), por ejemplo, compararon tres grupos de hombres y mujeres latinos, algunos de los cuales se habían incorporado del todo o parcialmente a una cultura nueva, y no encontraron invarianza de la escala, es decir, no hubo consistencia en los grupos de diferentes culturas ni entre sexos. En el estudio de Zhang, et al. (18), en el cual se evaluó la invarianza en dos poblaciones de adultos mayores diferentes desde el punto de vista social y cultural (población china y holandesa), se encontró que la escala no se había comportado de manera invariable en la población holandesa. En estos estudios, sin embargo, la varianza en el constructo se explicaría por una mayor cantidad de ítems, lo que necesariamente se refleja en una mayor varianza factorial (23).

Con los resultados obtenidos en el presente estudio, no es posible explicar si la invarianza de los perfiles se relacionaba con el contexto social, o si dichos resultados variarían significativamente al cambiar el modelo conceptual de la sintomatología depresiva, lo cual debería explorarse en futuros estudios.

Es necesario anotar algunas particularidades importantes del presente estudio, especialmente el hecho de que la pertenencia a un pueblo indígena no tiene una definición universal dada la diversidad existente a nivel mundial, por lo que hablar de la identidad de las poblaciones indígenas resulta complicado frente a la diversidad cultural, histórica, lingüística, de territorio, de costumbres y de adscripción de las propias personas a un grupo indígena $(28,29)$. De hecho, la definición de quién es y quién no es indígena es motivo de controversia actualmente, sobre todo en el marco jurídico y de los acuerdos internacionales, pues muchos de los derechos asociados con la pertenencia a un pueblo indígena dependen de su reconocimiento como tal (3).

En este estudio, se utilizó la lengua como un indicador de pertenencia a un grupo indígena, ya que este elemento se ha usado en encuestas mexicanas para la identificación de grupos indígenas (38). Sin embargo, se reconocen las limitaciones que ello entraña y el debate que suscita, pues se ha considerado que dicho criterio puede crear distinciones rígidas e irreales entre quién es indígena y quién no lo es. Debe reconocerse, además, la existencia de pueblos indígenas sin idiomas nativos, o que han sufrido la pérdida de su lengua, generalmente debido a las condiciones de pobreza, exclusión social y falta de participación política a las que se los ha sometido (3).

Por otro lado, el presente estudio tampoco permitió caracterizar diferentes comportamientos por grupos específicos de indígenas, al no haber sido una de las variables medidas en la ENSANUT 2012, y es probable que ello pudiera ocultar diferencias en ciertos grupo menos occidentalizados $(24,35,37)$. En todo caso, la muestra no hubiera permitido captar suficientes sujetos de estos subgrupos específicos.

Por último, debe reconocerse que solo se evaluó la varianza de configuración, o débil, y que para las aplicaciones en las que el objetivo es garantizar la equidad en salud de distintos grupos sociales o étnicos, se requiere la evaluación de la invariabilidad factorial estricta, así como del constructo latente en esta población (21). Sin embargo, el análisis de la varianza de configuración es el requisito básico de la invarianza factorial (21).

De todas maneras, este es un primer acercamiento a la evaluación de la invarianza factorial en la población indígena mexicana, en el cual se evidenció que la escala CES-D presentó invarianza factorial en los ocho perfiles de sexo, alfabetismo y la condición de hablante de una lengua indígena, considerados para el análisis, por lo que se sugiere su uso en encuestas y unidades primarias de atención en salud de la población de estudio, así como para el diagnóstico temprano y la identificación en estudios poblacionales. 
Si bien en diversos estudios se han detectado niveles, incluso elevados, de sintomatología depresiva en poblaciones indígenas en los últimos años $(39,40)$, y en algunos países, como Colombia y Canadá, se han encontrado altas tasas de suicidio en estos grupos $(41,42)$, los resultados de este estudio sugieren que entre los indígenas de México la sintomatología depresiva no es mayor que en otros grupos. Debe reconocerse, no obstante, el carácter exploratorio de estos datos, y la necesidad de mejorar la información estadística sobre salud mental en los grupos indígenas.

Los resultados de este trabajo no permiten concluir que la escala tiene invarianza factorial en todos los contextos, por lo que difícilmente puede extrapolarse a otras poblaciones fuera de México o, incluso, a comunidades más específicas dentro del país.

México es un país reconocido por su gran diversidad cultural y étnica, por lo tanto, resulta primordial considerar las características de la población indígena (39). En el país, la atención en salud general y mental no tiene un enfoque cultural diferenciado (35). Se ha determinado, además, que hay inequidades como consecuencia de las diferencias culturales, de costumbres y creencias, de religión y las debidas a la adscripción de las propias personas a un grupo indígena (25).

La detección de síntomas depresivos con un adecuado instrumento de tamización, permitiría mejorar las condiciones de salud de las poblaciones indígenas en regiones en donde no se presta adecuada atención a la salud mental, ya sea por los escasos recursos que se destinan para ello, o por la inexistencia de un sistema integrado de salud mental en la atención primaria. Es necesario, entonces, seguir recolectando información comprobada para garantizar el éxito de las políticas de atención en salud mental de las poblaciones indígenas orientadas a disminuir las inequidades a las que están sujetos estos grupos en un contexto multicultural como el de Latinoamérica.

\section{Conflicto de intereses}

Los autores declaran no tener conflicto de intereses.

\section{Financiación}

No se recibió ninguna fuente de financiación para el presente estudio.

\section{Referencias}

1. Ferrari AJ, Charlson FJ, Norman RE, Patten SB, Freedman G, Murray CJ, et al. Burden of depressive disorders by country, sex, age, and year: Findings from the Global Burden of Disease Study 2010. PLoS Med. 2013;10: 1-12. https://doi.org/10.1371/journal.pmed.1001547
2. Gracey $\mathbf{M}$, King $\mathbf{M}$. Indigenous health part 1: Determinants and disease patterns. Lancet. 2009;374:65-75. https://doi. org/10.1016/s0140-6736(09)60914-4

3. Banco Mundial. Latinomérica indígena en el siglo XXI. Informe. Primera década. Washington, D.C.: Banco Mundial; 2015. Fecha de consulta: $1^{\circ}$ de noviembre de 2015. Disponible en: http://bit.ly/2elbWMR

4. Asamblea General de Naciones Unidas. Programa de acción para el Segundo Decenio Internacional de los Pueblos Indígenas del Mundo: Informe del Secretario General. New York; 2005. Fecha de consulta: $1^{\circ}$ de noviembre de 2015. Disponible en: http://bit.ly/2fEhe1Z

5. Foro Internacional de Mujeres Indígenas. Estado de la cuestión sobre indicadores y pueblos indígenas en América Latina. En: VIII Encuentro Internacional de Estadísticas de Género para Políticas Públicas. Informe del Instituto Nacional de Estadística y Geografía. Fecha de consulta: 1 de noviembre de 2015. Disponible en: http://bit.ly/2eDOohu

6. Fuentealba RG, Bravo CA, Urrutia CM. Normas y punto de corte para la escala de depresión del Centro para Estudios Epidemiológicos (CES-D) en población juvenil chilena. Terapia Psicológica. 2015;22:145-56.

7. Consejo Nacional del Instituto Nacional de Lenguas Indígenas. Catálogo de las lenguas indígenas nacionales: variantes lingüísticas de México con sus autodenominaciones y referencias geoestadísticas. 2008. México: Diario Oficial de la Federación; 2008. p. 1-256.

8. División de Población del Centro Latinoamericano y Caribeño de Demografía. Salud de la población indígena en América Latina. Un panorama general. Primera edición. Santiago de Chile: Naciones Unidas; 2011. p. 81-94.

9. Lozano R, Gómez-Dantés H, Garrido-Latorre F, JiménezCorona A, Campuzano-Rincón JC, Franco-Marina F, et al. La carga de enfermedad, lesiones, factores de riesgo y desafíos para el sistema de salud en México. Salud Pública Mex. 2013;55:580-94.

10. Wancata J, Friedrich F. Depression: A diagnosis aptly used? Psychiatr Danub. 2015;23:406-11.

11. Coman EN, lordache E, Schensul JJ, Coiculescu I. Comparisons of CES-D depression scoring methods in two older adults ethnic groups. The emergence of an ethnicspecific brief three-item CES-D scale. Int J Geriatr Psychiatry. 2013;28:424-32. https://doi.org/10.1002/gps.3842

12. Radloff LS. The-CES-D-Scale: A self-report depression scale for research in the general population. Appl Psychol Meas. 1977;1:385-401. https://doi.org/10.1177/01466216 7700100306

13. Salinas-Rodríguez A, Manrique-Espinoza B, AcostaCastillo I, Téllez-Rojo MM, Franco-Núñez LA, GutiérrezRobledo LM, et al. Validación de un punto de corte para la escala de depresión del centro de estudios epidemiológicos, versión abreviada (CES-D-7). Salud Pública Mex. 2013;55:267-74.

14. Herrero J, Gracia E. Una medida breve de la sintomatología depresiva (CESD-7). Salud Ment. 2007;30:40-6.

15. Bojorquez C, Salgado-de Snyder N. Características psicométricas de la Escala Center for Epidemiological Studiesdepression (CES-D), versiones de 20 y 10 reactivos, en mujeres de una zona rural mexicana. Salud Ment. 2009;32: 299-307. 
16. Jia H, Chumbler N, Wang X, Chuang H, Damush T, Cameon R, et al. Racial and ethnic disparities in post-stroke depression detection. Int J Geriatr Psychiatry. 2010;25:298304. https://doi.org/10.1002/gps.2339

17. Yang L, Jia C-X, Qin P. Reliability and validity of the Center for Epidemiologic Studies Depression Scale (CES-D) among suicide attempters and comparison residents in rural China. BMC Psychiatry. 2015;15:76. https://doi.org/10.1186/ s12888-015-0458-1

18. Zhang B, Fokkema M, Cuijpers P, Li J, Smits N, Beekman A. Measurement invariance of the Center for Epidemiological Studies Depression Scale (CES-D) among Chinese and Dutch elderly. BMC Med Res Methodol. 2011;11:74. https://doi.org/10.1186/1471-2288-11-74

19. Fernández-Niño JA, Manrique-Espinoza BS, BojorquezChapela I, Salinas-Rodríguez A. Income inequality, socioeconomic deprivation and depressive symptoms among older adults in México. PLoS One. 2014;9:e108127. https:// doi.org/10.1371/journal.pone.0108127

20. Iwata N, Turner RJ, Lloyd DA. Race/ethnicity and depressive symptoms in community-dwelling young adults: A differential item functioning analysis. Psychiatry Res. 2002;110:281-9. https://doi.org/10.1016/S0165-1781(02)00102-6

21. Elosua P. Evaluación progresiva de la invarianza factorial entre las versiones original y adaptada de una escala de autoconcepto. Psicothema. 2005;17:356-62.

22. Meredith W, Teresi J. An essay on measurement and factorial invariance. Med Care. 2006;44(Suppl.3):S69-77. https://doi.org/10.1097/01

23. Rivera-Medina CL, Caraballo JN, Rodríguez-Cordero ER, Bernal G, Dávila-Marrero E. Factor structure of the CES-D and measurement invariance across gender for low-income Puerto Ricans in a probability sample. J Consult Clin Psychol. 2010;78:398-408. https://doi.org/10.1037/a0019054

24. Posner SF, Stewart AL, Marín G, Pérez-Stable EJ. Factor variability of the Center for Epidemiological Studies Depression Scale (CES-D) among urban Latinos. Ethn Health. 2001;6:137-44. https://doi.org/10.1080/1355785012 0068469

25. Tiburcio-Sainz M, Natera-Rey G. Adaptación al contexto ñahñú del Cuestionario de Enfrentamientos (CQ), la Escala de Síntomas (SRT) y la Escala de Depresión del Centro de Estudios Epidemiológicos (CES-D). Salud Ment. 2007;30:48-58.

26. Romero-Martínez M, Shamah-Levy T, Franco-Núñez A, Villalpando S, Cuevas-Nasu L, Gutiérrez JP, et al. Encuesta Nacional de salud y Nutrición 2012, diseño y cobertura. Salud Pública Mex. 2013;55(Supl. 2):S332-40.

27. Gutiérrez J, Rivera-Domaco J, Shamah-Levy T, Villalpando-Hernández S, Franco A, Cuevas-Nasu L, et al. Encuesta nacional de Salud y Nutrición (ENSANUT) 2012. Primera edición. Cuernavaca: Instituto Nacional de Salud Pública; 2012.

28. Centro de Información de las Naciones Unidas. Séptimo Periodo de Sesiones, Foro permanente para las cuestiones indígenas, 21 de abril al 2 de mayo de 2008. Organización de las Naciones Unidas. 2008. Fecha de consulta: 1 de abril de 2016. Disponible en: http://www.cinu.org.mx/prensa/ especiales/indigenas/\#5
29. Centro de Información de las Naciones Unidas. Quiénes son los pueblos indígenas. Quinta sesión del foro permanente para las cuestiones indígenas. Organización de las Naciones Unidas. 2007. Fecha de consulta: 1 de abril de 2016. Disponible en: http://bit.ly/2fDCrLo

30. Guillot G, Rousset F. Dismantling the Mantel tests. Methods Ecol Evol. 2013;4:336-44. https://doi.org/10.1111/2041-210x. 12018

31. Slone LB, Norris FH, Murphy AD, Baker CK, Perilla JL, Díaz D, et al. Epidemiology of major depression in four cities in México. Depress Anxiety. 2006;23:158-67. https:// doi.org/10.1002/da.20137

32. Rafful C, Medina-Mora ME, Borges G, Benjet C, Orozco R. Depression, gender, and the treatment gap in México. J Affect Disord. 2012;138:165-9. https://doi.org/10.1016/j. jad.2011.12.040

33. Kisely S, Alichniewicz KK, Black EB, Siskind D, Spurling G, Toombs M. The prevalence of depression and anxiety disorders in indigenous people of the Americas: A systematic review and meta-analysis. J Psychiatr Res. 2016;84:13752. https://doi.org/10.1016/j.jpsychires.2016.09.032

34. Angel R, Thoits $\mathbf{P}$. The impact of culture on the cognitive structure of illness. Cult Med Psychiatry. 1987;11:465-94. https://doi.org/10.1017/CBO9781107415324.004

35. Karasz A, García N, Ferri L. Conceptual models of depression in primary care patients: A comparative study. J Cross Cult Psychol. 2009;40:1041-59. https://doi.org/10. 1177/0022022109348782

36. Kirmayer LJ. Cultural variations in the clinical presentation of depression and anxiety: Implications for diagnosis and treatment. J Clin Psychiatry. 2001;62(Suppl.13):22-30.

37. Nguyen HT, Kitner-Triolo M, Evans MK, Zonderman AB. Factorial invariance of the CES-D in low socioeconomic status African Americans compared with a nationally representative sample. Psychiatry Res. 2004;126:177-87. https://doi.org/10.1016/j.psychres.2004.02.004

38. Yusim A, Anbarasan D, Hall B, Goetz R, Neugebauer $\mathbf{R}$, Stewart $\mathbf{T}$, et al. Sociocultural domains of depression among indigenous populations in Latin America. Int Rev Psychiatry. 2010;22:370-7. https://doi.org/10.3109/095402 61.2010 .500870

39. Cabada-Ramos E, Rincón-Cabada F, García-Cabrera $\mathrm{K}$, Munguía-Alamilla E, Torres-Iglesias E, MontoyaRodríguez M. Prevalencia de los síntomas de depresión posparto en población urbana, rural e indígena. Rev Esp Méd Quir. 2015;20:18-23.

40. Rey GN, Pérez FC, Barker S, Little TV, Aguilar PM. «Pa' qué sirvo yo, mejor me muero»: hacia la construcción de la percepción de sintomatología depresiva en una comunidad indígena. Salud Ment. 2012;35:63-70.

41. Instituto Nacional de Medicina Legal y Ciencias Forenses. Boletín epidemiológico. Suicidio en indígenas 2010-2014. Bogotá, D.C.: INMLCF; 2015.

42. Division of Social and Transcultural Psychiatry. The mental health of indigenous peoples. Montréal: McGill University; 2001. 